\title{
INJECTION CARBON STRIPPING FOIL ISSUES IN THE SNS ACCUMULATOR RING*
}

\author{
J. Beebe-Wang, Y. Y. Lee, D. Raparia, and J. Wei \\ Brookhaven National Laboratory, Upton, NY 11973, USA
}

\begin{abstract}
We are reporting the results of studies on issues related to the injection stripping foil in the Spallation Neutron Source (SNS) accumulator ring. The problems related to foil heating and foil lifetime, such as current density distribution and temperature distribution in the foil, are investigated. The impact of injection errors on the beam losses at the foil is studied. The particle traversal rate and the beam losses due to scattering in the foil are summarized. Finally, SNS end-to-end simulation results of the foil-missing rate, the foil-hitting rate and the maximum foil temperature are presented.
\end{abstract}

\section{INTRODUCTION}

One of the challenges for the SNS Project [1] is the multi-turn injection system of the accumulator ring. In the current design, $\mathrm{H}^{-}$particles are generated by the $\mathrm{H}^{-}$ion source, accelerated by the Linac and transported through the HEBT line to the injection point. At this location a charge exchange carbon foil of $200-400 \mu \mathrm{g} / \mathrm{cm}^{2}$ is used to strip two electrons off the incident $\mathrm{H}^{-}$particles. The protons will then circulate in the ring becoming a part of the accumulated beam. The particles that miss the foil or are not fully stripped in the foil will be further stripped through the injection dump foil system and be directed into the dump, becoming a part of a controlled beam loss.

Heating and beam losses are created at the foil due to the large impact of the initial hitting by $\mathrm{H}^{-}$and the multiple traversals by the circulating protons. We report simulation studies on this important issue. In our study, all of the physical quantities used in the simulations (table1) are chosen according to the design specification [1]. The initial foil-hit by each incident $\mathrm{H}^{-}$is counted as three to include the effects of two stripped electrons. Unless otherwise indicated, an average foil thickness of $300 \mu \mathrm{g} / \mathrm{cm}^{2}$ is assumed. The effect of space charge and magnet errors are not included in this study, since their impact on these foil issues are minimal. Their effects on the circulating beams are reported separately [2].

Table 1 Design parameters used in the simulations.

\begin{tabular}{|l|c|}
\hline Beam Kinetic Energy & $1 \mathrm{GeV}$ \\
\hline Protons per Pulse & $2.08 \times 10^{14}$ \\
\hline Stripping Foil Size & $6 \sigma_{\text {inj }}$ \\
\hline Horizontal tune $v_{x} /$ Vertical tune $v_{y}$ & $6.3 / 5.8$ \\
\hline Injected beam Emittance & $0.5 \pi \mathrm{mm}-\mathrm{mr}$ \\
\hline Un-norm. Painted beam Emittance & $120 \pi \mathrm{mm}-\mathrm{mr}$ \\
\hline
\end{tabular}

*Work performed under the auspices of the U. S. Department of Energy

\section{FOIL HEATING}

The foil temperature distribution is governed by the current density distribution on the foil during injection. Different painting schemes produce not only different final particle distributions, but also generate different foil traversal patterns and therefore different current density distributions in the foil. There are two basic painting schemes - correlated and anti-correlated painting incorporated in the SNS accumulator ring design [3].

In this study the current density distributions are simulated through turn-by-turn beam tracking during correlated or anti-correlated injection painting. The foil temperature distributions are then converted from the current density distributions through the relationship presented in figure 1. This is deduced from the model described in reference [4] with a foil thickness of $300 \mu \mathrm{g} / \mathrm{cm}^{2}$. The model includes (1) the radiation heat transfer between the carbon foil and the stainless steel beam pipe, (2) the heat conduction through the foil to its base, (3) a natural convection condition on the outer surface of the beam pipe, and (4) a Gaussian injected beam distribution. Figure 2 and figure 3 show, for the same injected beam distribution, the foil temperature distribution resulting from correlated and anti-correlated painting respectively.

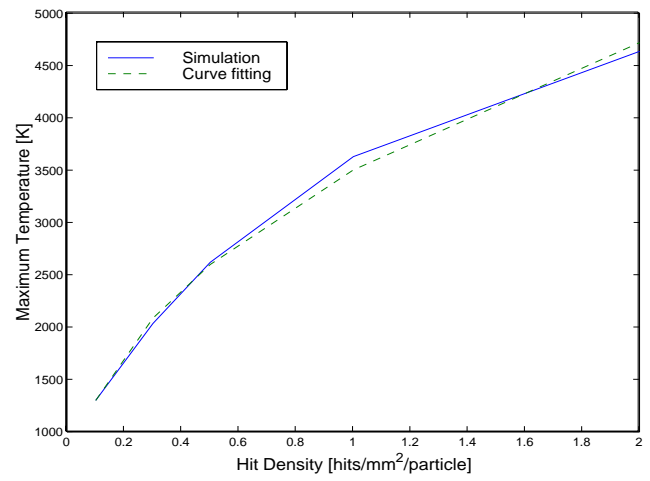

Figure 1 Foil temperature vs. beam current density (blue line) deduced from the model [4] and the curve fit (green line) used in foil temperature distribution studies.

In general, due to the nature of the painting schemes, the maximum foil temperature from an anti-correlated painting is higher (2378K in this study) than the one from correlated painting $(2243 \mathrm{~K})$. The foil lifetime tests at BNL Linac show that maximum single foil life time is $\sim 78$ hours and decrease sharply when the foil temperature exceeds $2500 \mathrm{~K}$ [5]. At BNL, a multiple-foil exchange 
system has been designed in order to extend the noninteracted operation time with the limited foil lifetime.

The maximum foil temperature is also a function of the emittance of the injected beam. For a given foil thickness, the maximum foil temperature decreases while the Linac beam emittance increases. However, the foilhitting rate also increases, if a foil size of $6 \sigma_{\text {inj }}$ is kept, which will increase uncontrolled beam losses discussed later in section 3.2. figure 4 shows: (a) the maximum foil temperature and (b) the foil traversal rate as functions of normalized RMS emittance of injected beam.
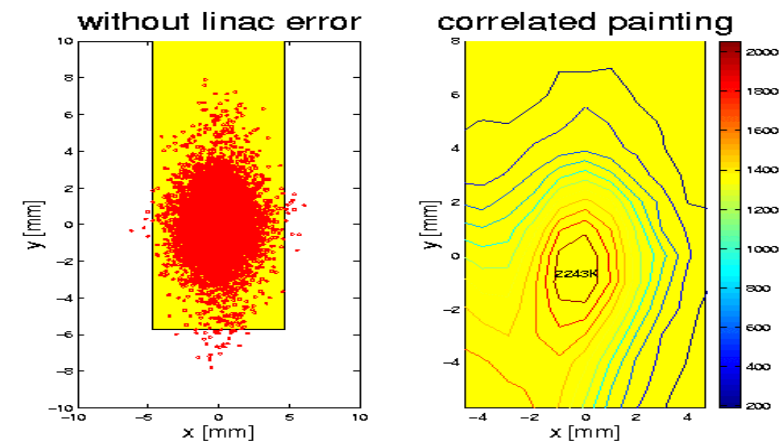

Figure 2 Computer simulation results of (a) Injected beam distribution on the foil and (b) the foil temperature distribution due to correlated painting.
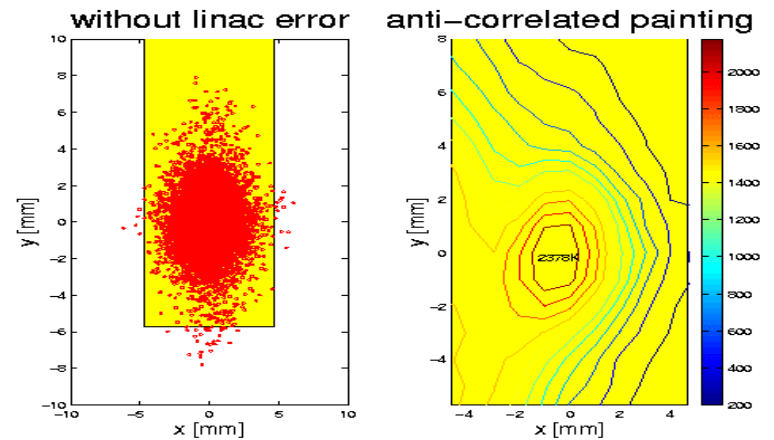

Figure 3 Computer simulation results of (a) Injected beam distribution on the foil and (b) the temperature distribution in the foil due to anti-correlated painting.
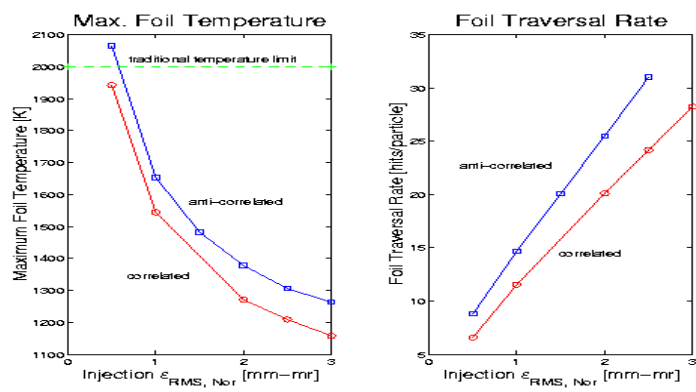

Figure 4 (a) the maximum foil temperature and (b) foil traversal rate as functions of normalized RMS emittance of injected beam. The red and blue lines indicate the simulation results obtained with correlated and anticorrelated paintings respectively.

\section{BEAM LOSSES AT INJECTION FOIL}

There are two kinds of beam losses at the SNS injection stripping foil: (1) controlled losses which can be directed to the injection beam dump located down stream of the injection foil; and (2) uncontrolled losses which are scattered in all directions and generate radiation in the injection area. For a high intensity storage ring, such as the SNS accumulator, there are very stringent limitations on both kinds of beam losses. Therefore, investigations are necessary in order to estimate the beam losses.

\subsection{Controlled Beam Losses}

The major sources of controlled beam losses are (1) injected particles missing the foil and (2) foil inefficiency. The injection errors of the center location and the emittance of the Linac beams are the two major contributors to particles missing the foil. Figure 5 shows the geometrical foil-missing rate vs. injection position error $\Delta \mathrm{x}, \Delta \mathrm{y}$ and Linac beam emittance error $\Delta \varepsilon_{\mathrm{x}}, \Delta \varepsilon_{\mathrm{y}}$.

In addition, during injection a fraction of injected $\mathrm{H}^{-}$ particles do not miss the foil but pass through the foil without been stripped or deflected. This loss is characterized by "foil inefficiency" which is a function of foil thickness. It is estimated that for foil thickness of 200$400 \mu \mathrm{g} / \mathrm{cm}^{2}$ the foil inefficiency is $2-10 \%$. These unstripped $\mathrm{H}^{-}$particles, joined by the $\mathrm{H}^{-}$particles missing the
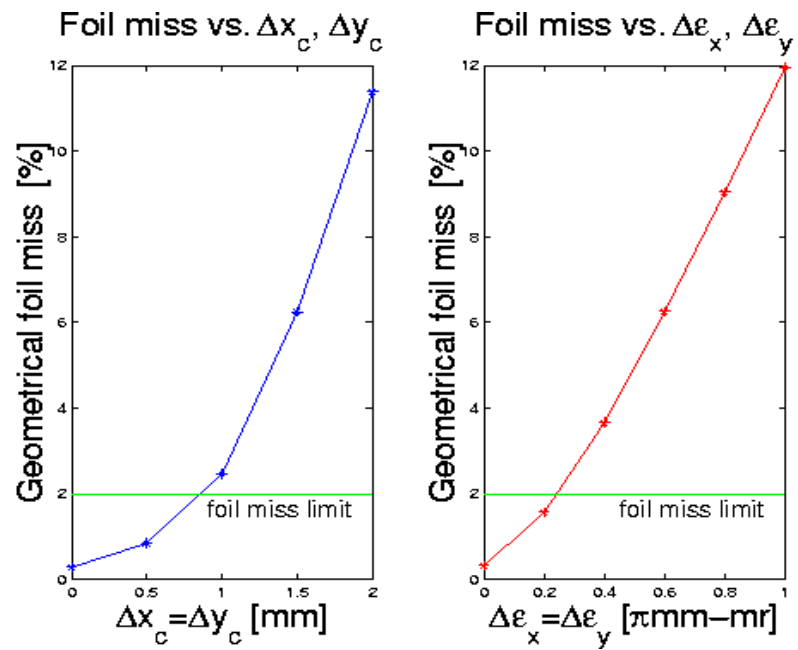

foil, are directed to the injection beam dump.

The maximum amount of the beam that the SNS injection dump can handle is $10 \%$ of injected beam at its full intensity. This limits the total controlled loss due to foil miss and foil inefficiency to be $<10 \%$. So, if the operation is at an average foil inefficiency of $\sim 6 \%$, the total geometrical foil missing rate will be limited to $\sim 4 \%$ which translates into an injection error limitation of $\Delta \mathrm{x}=\Delta \mathrm{y}=1 \mathrm{~mm}$ and $\Delta \varepsilon_{\mathrm{x}}=\Delta \varepsilon_{\mathrm{y}}=0.2 \pi \mathrm{mm}-\mathrm{mr}$ as indicated by the green lines in figure 5 .

Figure 5 Controlled beam losses due to injection errors. (a) geometrical foil-missing rate vs. injection point error $\Delta \mathrm{x}=\Delta \mathrm{y}$, and (b) geometrical foil-missing rate vs. injection beam emittance error $\Delta \varepsilon_{\mathrm{x}}=\Delta \varepsilon_{\mathrm{y}}$. 


\subsection{Uncontrolled Beam Losses}

As a consequence of particle traversal in the stripping foil, there are beam losses at the foil through mechanisms of (1) nuclear scattering, (2) energy straggling and (3) multiple scattering.

\subsubsection{Nuclear Scattering}

The beam loss due to nuclear scattering in the foil is a function of the foil traversal rate, foil thickness and Linac beam emittance. For given painting schemes (correlated and anti-correlated) and given stripping foil thickness $\left(200 \mu \mathrm{g} / \mathrm{cm}^{2}\right.$ and $\left.400 \mu \mathrm{g} / \mathrm{cm}^{2}\right)$, figure $6(\mathrm{a})$ shows the fractional nuclear scattering losses as a function of the normalized RMS Linac beam emittance [6]. If all of these losses are located in the injection straight section $(\sim 10 \mathrm{~m})$, the resulting estimated radiation level, at 1 foot from the beam line after a 100-day run followed by 4 hours of shutdown, as a function of Linac emittance can be seen in figure 6 [6]. For the current SNS design, the foil thickness is $300 \mu \mathrm{g} / \mathrm{cm}^{2}$ and the normalized RMS linac beam emittance is $0.5 \pi \mathrm{mm}-\mathrm{mr}$. The estimated fractional beam loss due to nuclear scattering is $3 \times 10^{-5}$.
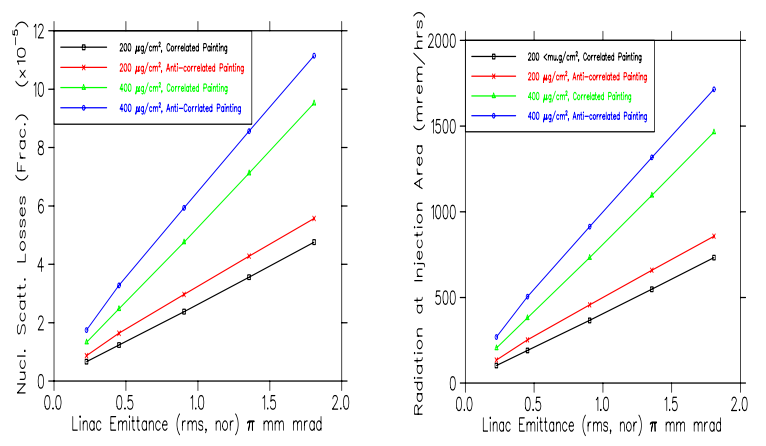

Figure 6 (a) Fractional beam losses at injection area and (b) radiation level due to nuclear scattering in the stripping foil as functions of normalized RMS emittance of injected beam [6].

\subsubsection{Energy Straggling}

As particles traverse through the injection foil, a fraction of their energies are lost and deposited in the foil. Some of the circulating protons, which lie in the tail of Landau distribution, will go through large synchrotron oscillations and may end up in the beam gap [7]. These protons will be lost at the time of extraction or at a dispersive location in the ring. The estimated fractional beam loss in the gap due to energy straggling is $3 \times 10^{-6}$ for a foil thickness of $300 \mu \mathrm{g} / \mathrm{cm}^{2}$ and normalized RMS Linac beam emittance of $0.5 \pi \mathrm{mm}-\mathrm{mr}$.

\subsubsection{Multiple Scattering}

The major effect of particle multiple scattering in the foil is to increase the transverse beam emittance. For a foil thickness of $300 \mu \mathrm{g} / \mathrm{cm}^{2}$ and normalized RMS linac beam emittance of $0.5 \pi \mathrm{mm}-\mathrm{mr}$, the estimated transverse emittance growth due is $4 \times 10^{-2} \pi \mathrm{mm}-\mathrm{mr}$.

\section{END-TO-END SIMULATION}

Table 2 list a more realistic foil missing-rate, foil hitting-rate and maximum foil temperature obtained from computer simulations with 11 sets of injected particle distributions.

Table 2 Foil missing-rate, foil hitting-rate and maximum foil temperature from SNS end-to-end simulations.

\begin{tabular}{|c|c|c|c|c|c|}
\hline Linac & Foil Miss & \multicolumn{2}{|c|}{ Foil Traveral Rate } & \multicolumn{2}{c|}{ Max. Foil Temp. (K) } \\
\cline { 3 - 6 } Error & $(\%)$ & correlated & anti-corr. & correlated & anti-corr. \\
\hline no error & 0.485 & 6.51 & 8.66 & 2243 & 2378 \\
\hline set \#1 & 1.553 & 6.17 & 8.64 & 2185 & 2248 \\
\hline set \#2 & 1.338 & 6.86 & 8.99 & 2273 & 2359 \\
\hline set \#3 & 0.378 & 8.33 & 10.06 & 2205 & 2282 \\
\hline set \#4 & 0.637 & 8.18 & 10.45 & 2190 & 2284 \\
\hline set \#5 & 0.442 & 6.93 & 8.66 & 2167 & 2376 \\
\hline set \#6 & 0.529 & 7.08 & 9.29 & 2189 & 2336 \\
\hline set \#7 & 0.583 & 6.59 & 9.05 & 2207 & 2288 \\
\hline sat \#8 & 1.413 & 5.92 & 7.99 & 2113 & 2282 \\
\hline set \#9 & 0.744 & 6.21 & 8.79 & 2139 & 2279 \\
\hline set \#10 & 0.658 & 7.09 & 9.178 & 2153 & 2319 \\
\hline average & 0.796 & 6.89 & 9.08 & 2188 & 2312 \\
\hline
\end{tabular}

\section{DISCUSSION AND CONCLUSION}

Our investigation has shown that foil lifetime and beam losses at the foil are important issues for the SNS injection. They could become the major limitations to the continuous running and hands-on maintenance during SNS future operations. The limitations of controlled and uncontrolled beam losses at the foil impose very high requirements on the quality and stability of Linac beams. Development of alternative carbon foil materials is desirable. At BNL/SNS, together with Fiber Materials Inc., many carbon materials have been developed, treated and tested [5]. Further efforts are continuously being made to prolong stripping foil lifetime.

\section{REFERENCES}

[1] J. Wei et al, "Low-Loss Design for the High-Intensity Accumulator Ring of the Spallation Neutron Source", Accepted for publication in PRST-AB, 2000.

[2] J. Beebe-Wang, A. V. Fedotov and J. Wei, "Space Charge and Magnet Errors Simulations for the SNS Accumulator Ring" Proceedings of the EPAC 2000, Vienna, Austria, 26-30 June 2000, p.1286-1288.

[3] J. Beebe-Wang, Y. Y. Lee, D. Raparia, J. Wei, J., C. R. Prior, and S. Machida, "Beam Properties in the SNS Accumulator Ring Due to Transverse Phase Space Painting", Proceedings of the EPAC 2000, Vienna, Austria, 26-30 June 2000, p.1465-1467.

[4] C. J. Liaw, Y.Y. Lee, J. Alessi and J. Tuozzolo, "Calculation of Maximum Temperature on the Carbon Stripping Foil of the Spallation Neutron Source", Proceedings of the PAC99, New York, USA, March 27- April 2, 1999.

[5] C. J. Liaw, Y.Y. Lee, J. and J. Tuozzolo, "Life Time of Carbon Stripping Foils For the Spallation Neutron Source" these proceedings.

[6] D. Raparia, J. Alessi, Y.Y. Lee, J. Wei and W.T. Weng, "Impact of the Superconducting Linac on the HEBT and Ring", BNL/SNS Tech Note No.070, January, 2000.

[7] D. Raparia, "Estimated Beam Loss due to Energy Straggling through the Charge Exchange Injection Foil", BNL/SNS Tech Note No.058, February, 1999. 